\title{
MEASURES IN BOOLEAN ALGEBRAS
}

\author{
BY \\ ALFRED HORN AND ALFRED TARSKI
}

Introduction. By a measure on a Boolean algebra we understand as usual a function defined over all elements of the algebra, assuming finite non-negative real numbers as values (but not identically vanishing), and satisfying the condition of finite additivity: the function value at a sum of two disjoint elements equals the sum of the function values at these elements. By including in this definition some additional conditions we arrive at special kinds of measures: two-valued measures, strictly positive measures, and countably additive measures.

In $\$ 1$ we concern ourselves with the general notion of measure; we show how a measure-and in particular a two-valued measure-defined on a subalgebra of a given Boolean algebra, or even on an arbitrary subset of the algebra, can be extended to the whole algebra. In $\$ \S 2$ and 3 we consider special kinds of measures, in fact, strictly positive and countably additive measures, and we establish several partial criteria (necessary or sufficient conditions) for their existence. Some of the results stated in this paper can be obtained in a roundabout way from what is to be found in the literature, but they will be provided here with rather simple and direct proofs, without applying notions not involved in the formulation of the results.

Terminology and symbolism( ${ }^{1}$. Given any two sets $A$ and $B$, we denote their union (or sum) by $A \cup B$, their intersection (or product) by $A \cap B$, their difference by $A-B$. The symbol $\subseteq$ (or $\supseteq$ ) is used to denote set inclusion, $\in$ the membership relation, and $\{a\}$ the set consisting of just one element a. The symbol

$$
\underset{x}{\mathrm{E}}[\cdots]
$$

will denote the set of all elements $x$ which satisfy the condition formulated in square brackets. The $n$-termed sequence with the terms $a_{0}, \cdots, a_{n-1}$ is denoted by $\left\langle a_{0}, \cdots, a_{n-1}\right\rangle$ (notice that the sequence begins with $a_{0}$, and not with $a_{1}$ ); analogous symbolism is used for infinite sequences.

"Countable" is used here in the sense of "finite or denumerably infinite." $\boldsymbol{\aleph}_{0}$ denotes the power of the set of natural numbers, $\boldsymbol{\aleph}_{1}$ the power of the set of ordinal numbers of the second class, and $c$ the power of the set of real numbers.

Presented to the Society, November 29, 1947 ; received by the editors September 29, 1947.

(1) For set-theoretical notions involved in this discussion consult Hausdorff [1] and Sierpinski [1]. For notions of general algebraic nature and those applying specifically to Boolean algebras consult Birkhoff [1]. Numbers in brackets refer to the bibliography at the end of this paper. 
A nonempty family of subsets of a given set $U$ which is closed under finite set-theoretical addition and under complementation (with respect to $U$ ) is referred to in this paper as a field of sets. A field $F$ is called countably complete if it is closed under countable addition of sets; it is called countably complete in the wider sense if it satisfies the following condition: whenever for an infinite sequence of sets $S_{0}, S_{1}, \ldots$ in $F$ there is a smallest set $S$ in $F$ including all of them, this set $S$ coincides with the sum of the sets $S_{0}, S_{1}, \cdots$ Obviously, every countably complete field of sets is also countably complete in the wider sense.

The notion of a set which is (partially, simply, or well) ordered by a given relation $\prec$ and the notion of two similarly ordered sets are assumed to be known. By an interval of an ordered set $S$ we understand here what is sometimes called a half-open interval, that is, a set of the form

$$
\begin{gathered}
\underset{x}{\mathrm{E}}[x \in S, x \neq a, a<x<b] \quad \text { or } \underset{x}{\mathrm{E}}[x \in S, x \neq a, a \prec x] \text { or } \\
\underset{x}{\mathrm{E}}[x \in S, x<b]
\end{gathered}
$$

where $a$ and $b$ are arbitrary elements of $S$. A subset $D$ of $S$ is said to be dense in $S$ if every nonempty interval of $S$ contains at least one element of $D$.

A function $f$ whose domain (the set of argument values) coincides with a given set $S$ is referred to as a function on $S$. On the other hand we say that the functions $f$ and $g$ agree on a set $S$ if $S$ is a subset of the domains of both functions, and if $f(x)=g(x)$ for every $x$ in $S$.

By a Boolean algebra we shall understand as usual a system formed by a set $A$ of arbitrary elements $a, b, c, \cdots$ and by the fundamental operations of addition (join operation) + , multiplication (meet operation) $\cdot$, and complemention -, which are assumed to satisfy certain familiar postulates. To simplify the symbolism we shall not distinguish between a Boolean algebra and the set of all its elements. We assume to be known how, in terms of the fundamental operations, other Boolean algebraic notions can be definedsuch as the elements 0 and 1 , the relation of inclusion $\leqq$ (or $\geqq$ ), that of strict inclusion $<$ (or $>$ ), the sum $\sum$ and the product $\Pi$ of an arbitrary system of elements (in particular, of a finite or infinite sequence). It should be noticed that the symbols $+, \cdot, 0,1$, and so on, will also be used in their ordinary arithmetical meaning, in application to natural and real numbers; the meaning in which a symbol is used will always be clear from the context.

The notions of general algebraic nature-such as subalgebra, ideal, the quotient algebra $A / I$ (of an algebra $A$ over an ideal $I$ ), direct product, and isomorphism-are familiar from the literature; the same applies to the more special notions of an atom, an atomistic and an atomless algebra, a complete and a countably complete algebra.

A set $D$ of nonzero elements of a Boolean algebra $A$ is said to be dense in $A$ if for every element $x$ in $A, x \neq 0$, there is an element $y$ in $D$ which is in- 
cluded in $x(y \leqq x)$; this is equivalent to saying that every element $x$ in $A$ is the sum of all elements $y$ in $D$ which are included in $x$. A Boolean algebra $A$ is called separable if there is a countable set $D$ which is dense in $A$.

A Boolean algebra $A$ is called countably distributive if the following condition is satisfied: Let $N$ be the set of all infinite sequences $n=\left\langle n_{0}, n_{1}, \cdots\right\rangle$ of natural numbers. Given any double sequence of elements $a_{i, j}$ in $A, i, j$ $=0,1, \cdots$, if all the sums $\sum_{j<\infty} a_{i, j}$ for $i<\infty$, their product $\prod_{i<\infty} \sum_{j<\infty} a_{i, j}$, and all the products $\prod_{i<\infty} a_{i, n_{i}}$ for $n \in N$ exist, then the sum $\sum_{n \in N} \prod_{i<\infty} a_{i, n_{i}}$ also exists, and we have

$$
\prod_{i<\infty} \sum_{j<\infty} a_{i, j}=\sum_{n \in N} \prod_{i<\infty} a_{i, n_{i}}
$$

If the formula just mentioned is only assumed to hold for those double sequences of elements $a_{i, j}$ which satisfy the condition $a_{i, j} \leqq a_{i, j+1}$ for $i, j$ $=0,1, \cdots$, the algebra is called weakly countably distributive.

When referring to a Boolean algebra of sets we shall always understand that set-theoretical addition, multiplication, and complementation (with respect to a fixed set) are taken as fundamental operations. As is well known, a family of sets is a Boolean algebra in this sense if and only if it is a field of sets. If, in particular, $S$ is a set which is simply ordered by a relation $\prec$, then all the finite sums of intervals of $S$ constitute a Boolean algebra, which will be referred to as the interval algebra of $S$ (under $<$ ).

1. Measure and partial measure. Throughout this section the symbol $A$ will be used to denote an arbitrary Boolean algebra (and in most cases the assumption that $A$ is a Boolean algebra will not be explicitly stated).

Definition 1.1. A function $f$ on a Boolean algebra $A$ is called a measure on $A$ if it is a real-valued function such that

(i) $0 \leqq f(x)$ for $x \in A$,

(ii) $f(x+y)=f(x)+f(y)$ whenever $x, y \in A$ and $x \cdot y=0$,

(iii) $f(1)=1$.

Condition (iii) in this form is not essential for our purpose. Evidently any function, not assuming $\infty$ as a value, which satisfies all the conditions of 1.1 except for (iii) and which is not identically 0 can be converted into a measure by dividing by $f(1)$.

CoROllary 1.2. If $f$ is a measure on $A$, then

(i) $f(0)=0$,

(ii) $f(x) \leqq f(y)$ whenever $x, y \in A$ and $x \leqq y$.

Proof. This follows from 1.1 .

Corollary 1.3. If $f$ is a measure on $A$, and $a_{0}, \cdots, a_{m-1} \in A$, then

$$
\sum_{k<m} f\left(a_{k}\right)=\sum_{k<m} f\left(\sum_{p \in S^{m, k}} \prod_{i \leqq k} a_{p_{i}}\right)
$$


where $S^{m, k}$ is the set of all sequences $p=\left\langle p_{0}, \cdots, p_{k}\right\rangle$ with $0 \leqq p_{0}<\cdots<p_{k}$ $<m$.

Proof. We proceed by induction. For $m=0,1$ the conclusion is obvious. For $m=2$ it reduces to the well known formula

$$
f\left(a_{0}\right)+f\left(a_{1}\right)=f\left(a_{0}+a_{1}\right)+f\left(a_{0} \cdot a_{1}\right),
$$

which results from 1.1. Using this formula we pass from $m=2$ to $m=3$ as follows:

$$
\begin{aligned}
f\left(a_{0}\right)+f\left(a_{1}\right)+f\left(a_{2}\right)= & f\left(a_{0}+a_{1}\right)+f\left(a_{0} \cdot a_{1}\right)+f\left(a_{2}\right) \\
= & f\left(a_{0}+a_{1}+a_{2}\right)+f\left(a_{0} \cdot a_{2}+a_{1} \cdot a_{2}\right)+f\left(a_{0} \cdot a_{1}\right) \\
= & f\left(a_{0}+a_{1}+a_{2}\right)+f\left(a_{0} \cdot a_{1}+a_{0} \cdot a_{2}+a_{1} \cdot a_{2}\right) \\
& +f\left(a_{0} \cdot a_{1} \cdot a_{2}\right) .
\end{aligned}
$$

The passage from $m$ to $m+1$ is analogous.

It is well known that measures exist on an arbitrary Boolean algebra $A$. What is perhaps more important, a measure on a subalgebra of $A$ can always be extended to a measure on $A$ (obviously, 1.1 applies automatically to any subalgebra $B$ of $A$, since $B$ is itself a Boolean algebra with the same fundamental operations as $A$ ). We shall establish this fact in an even stronger form by considering, instead of subalgebras, arbitrary subsets of $A$ containing the element $1\left({ }^{2}\right)$. This will require an appropriate extension of 1.1 ; measures on arbitrary subsets will be referred to as partial measures (see 1.7 below). To discuss these partial measures we need a Boolean algebraic notion which is not familiar from the literature:

DeFINITION 1.4. Let $a_{0}, \cdots, a_{m-1}$ and $b_{0}, \cdots, b_{n-1}$ be arbitrary elements of $A$. We agree to say that

if

$$
\begin{gathered}
\left\langle a_{0}, \cdots, a_{m-1}\right\rangle \leqq\left\langle b_{0}, \cdots, b_{n-1}\right\rangle \\
\sum_{p \in S^{m, k}} \prod_{i \leqq k} a_{p_{i}} \leqq \sum_{p \in S^{n, k}} \prod_{i \leqq k} b_{p_{i}} \text { for every } k<m,
\end{gathered}
$$

where $S^{r, k}(r=m, n)$ has the same meaning as in 1.3 .

We list without proof some elementary properties of the relation just defined.

(2) This result was established in a more abstract and general form in Tarski [1]. (To apply the results of that paper to a Boolean algebra, the latter should be treated as a system with a fundamental operation which coincides with the usual Boolean algebraic addition when applied to two disjoint elements, but which is left undefined otherwise. Boolean algebras in this conception do not satisfy the postulates of $\$ 1$ of the paper in question, but the desired result can be obtained by applying the remarks in $\$ 2$ of that paper.) In this section we follow the general argument of Tarski [1], but we modify it so as to make it apply directly to Boolean algebras; due to specific properties of these algebras, the argument undergoes simplification in various places. 
Theorem 1.5. Let $a_{0}, \cdots, a_{m-1}, b_{0}, \cdots, b_{n-1}, c_{0}, \cdots, c_{p-1}, d_{0}, \cdots, d_{q-1}$ be arbitrary elements of $A$. We then have:

(i) If $m=n$ and $\left\langle b_{0}, \cdots, b_{n-1}\right\rangle$ is a permutation of $\left\langle a_{0}, \cdots, a_{m-1}\right\rangle$, then

$$
\left\langle a_{0}, \cdots, a_{m-1}\right\rangle \leqq\left\langle b_{0}, \cdots, b_{n-1}\right\rangle \text {. }
$$

(ii) If

$$
\left\langle a_{0}, \cdots, a_{m-1}\right\rangle \leqq\left\langle b_{0}, \cdots, b_{n-1}\right\rangle \leqq\left\langle c_{0}, \cdots, c_{p-1}\right\rangle
$$

then

$$
\left\langle a_{0}, \cdots, a_{m-1}\right\rangle \leqq\left\langle c_{0}, \cdots, c_{p-1}\right\rangle
$$

(iii) If

$$
\left\langle a_{0}, \cdots, a_{m-1}\right\rangle \leqq\left\langle b_{0}, \cdots, b_{n-1}\right\rangle
$$

and

$$
\left\langle c_{0}, \cdots, c_{p-1}\right\rangle \leqq\left\langle d_{0}, \cdots, d_{q-1}\right\rangle
$$

then

$$
\left\langle a_{0}, \cdots, a_{m-1}, c_{0}, \cdots, c_{p-1}\right\rangle \leqq\left\langle b_{0}, \cdots, b_{n-1}, d_{0}, \cdots, d_{q-1}\right\rangle .
$$

(iv) If

$$
\left\langle a_{0}, \cdots, a_{m-1}\right\rangle \leqq\left\langle b_{0}, \cdots, b_{n-1}\right\rangle \text { and } a_{m-1}=b_{n-1},
$$

then

$$
\left\langle a_{0}, \cdots, a_{m-2}\right\rangle \leqq\left\langle b_{0}, \cdots, b_{n-2}\right\rangle .
$$

(v) $\left\langle a_{0}, \cdots, a_{m-1}\right\rangle \leqq\left\langle a_{0}, \cdots, a_{m-1}, 0\right\rangle \leqq\left\langle a_{0}, \cdots, a_{m-1}\right\rangle$.

(vi) If

$$
\left\langle a_{0}, \cdots, a_{m-1}\right\rangle \leqq\left\langle b_{0}, \cdots, b_{n-1}\right\rangle
$$

and $b_{n-1} \cdot a_{i}=0$ for all $i<m$, then

$$
\left\langle a_{0}, \cdots, a_{m-1}\right\rangle \leqq\left\langle b_{0}, \cdots, b_{n-2}\right\rangle .
$$

(vii) If

$$
b_{k}=\sum_{p \in S^{m, k}} \prod_{i \leqq k} a_{p_{i}} \quad \text { for } k<m,
$$

$S^{m, k}$ having the same meaning as in 1.3, then

$$
\left\langle a_{0}, \cdots, a_{m-1}\right\rangle \leqq\left\langle b_{0}, \cdots, b_{m-1}\right\rangle \leqq\left\langle a_{0}, \cdots, a_{m-1}\right\rangle .
$$

DEFINITION 1.6. A real-valued function $f$ on a subset $S$ of a Boolean algebra $A$ is called a partial measure on $S$ if the following conditions are satisfied:

(i) $0 \leqq f(x)$ for $x \in S$. 
(ii) If $a_{0}, \cdots, a_{m-1}, b_{0}, \cdots, b_{n-1} \in S$ and

$$
\left\langle a_{0}, \cdots, a_{m-1}\right\rangle \leqq\left\langle b_{0}, \cdots, b_{n-1}\right\rangle \text {, }
$$

then

$$
\sum_{i<m} f\left(a_{i}\right) \leqq \sum_{j<n} f\left(b_{j}\right)
$$

(iii) $1 \in S$ and $f(1)=1$.

Definition 1.6 could be extended to subsets of $A$ which do not contain 1 as an element. However, the definition would have to assume a more involved form and our further discussion would undergo some complication.

Corollary 1.7. If $1 \in S \subseteq T$, $f$ is a function on $S$, and $g$ is a partial measure on $T$ which agrees with $f$ on $S$, then $f$ is a partial measure on $S$.

Proof. This follows from 1.6.

CoROllary 1.8. Let $f$ be a partial measure on $S$.

(i) If $0 \in S$, then $f(0)=0$.

(ii) If $x, y \in S$ and $x \leqq y$, then $f(x) \leqq f(y)$.

(iii) If $x, y, x+y \in S$ and $x \cdot y=0$, then $f(x+y)=f(x)+f(y)$.

(iv) If $x, y, x+y, x \cdot y \in S$, then $f(x+y)+f(x \cdot y)=f(x)+f(y)$.

Proof. To obtain (ii) we notice that if $x \leqq y$, we have by $1.3\langle x\rangle \leqq\langle y\rangle$, and hence, by 1.6, $f(x) \leqq f(y)$. Similarly, under the hypotheses of (iii) [or (iv)], we have, by 1.5 (vii) and $1.5(\mathrm{v})$,

$$
\langle x, y\rangle \leqq\langle x+y\rangle \leqq\langle x, y\rangle \quad[\text { or }\langle x, y\rangle \leqq\langle x+y, x \cdot y\rangle \leqq\langle x, y\rangle],
$$

and hence by 1.6 the conclusion. Finally, (i) obviously follows from (iii).

THEOREM 1.9. (i) Let $S$ be a subset of $A$ closed under the operations of addition and multiplication. For a real-valued function $f$ on $S$ to be a partial measure it is necessary and sufficient that f and $S$ satisfy 1.6 (i), 1.6 (iii), 1.8 (i), 1.8 (ii), and 1.8 (iv).

(ii) Let $S$ be a subalgebra of $A$ (or, in particular, let $S=A$ ). For a function $f$ to be a partial measure on $S$, it is necessary and sufficient that $f$ be a measure on $S$.

Proof. By 1.6 and 1.8, the conditions stated in (i) are necessary for $f$ to be a partial measure on $S$. To show that these conditions are also sufficient, we first derive 1.3 (i) from 1.8 (iv) by arguing as in the proof of 1.3 , and then obtain 1.6 (ii) with the aid of 1.5 (vii), 1.8 (i), and 1.8 (ii). Statement (ii) follows from (i) by 1.1, 1.2, 1.3, and 1.8 (iii).

THEOREM 1.10. Let $f$ be a function on $S_{0} \cup S_{1} \subseteq A$ such that $f(x)=0$ for $x \in S_{0}$ and $f(x)=1$ for $x \in S_{1}$. Then $f$ is a partial measure on $S_{0} \cup S_{1}$ if and only if $1 \in S_{1}$ and 


$$
\sum_{i<m} a_{i}+\sum_{j<n} \overline{b_{j}} \neq 1
$$

whenever $a_{i} \in S_{0}, b_{i} \in S_{1}$ for $i<m, j<n$, and $m, n<\infty$.

Proof. Let $f$ be a partial measure on $S_{0} \cup S_{1}$. Then, by 1.6 (iii), $1 \in S_{1}$. Suppose

$$
\sum_{i<m} a_{i}+\sum_{j<n} \overline{b_{j}}=1
$$

for some choice of elements $a_{i} \in S_{0}, b_{j} \in S_{1}$. Then

$$
\prod_{j<n} b_{j} \leqq \sum_{i<m} a_{i}
$$

This implies

$$
\left\langle b_{0}, \cdots, b_{n-1}\right\rangle \leqq\left\langle a_{0}, \cdots, a_{m-1}, 1, \cdots, 1\right\rangle
$$

where 1 is repeated $n-1$ times in the sequence on the right. By 1.6 (ii) we have the absurdity $n \leqq n-1$.

Conversely, suppose $1 \in S_{1}$ and assume (i) holds. To show $f$ is a measure we need only verify 1.6 (ii). Suppose

$$
\left\langle b_{0}, \cdots, b_{n-1}, c_{0}, \cdots, c_{p-1}\right\rangle \leqq\left\langle d_{0}, \cdots, d_{q-1}, a_{0}, \cdots, a_{m-1}\right\rangle
$$

where $b_{i}, d_{i} \in S_{1}$ and $a_{i}, c_{i} \in S_{0}$. If we had

$$
\sum_{i<n} f\left(b_{i}\right)+\sum_{i<p} f\left(c_{i}\right)>\sum_{i<q} f\left(d_{i}\right)+\sum_{i<m} f\left(a_{i}\right)
$$

we should have $n>q$. By applying 1.4 to (1), we obtain a sequence of inequalities involving sums of products of $k$ elements. Combined with $n>q$, the inequality for $k=n$ would imply

$$
\prod_{j<n} b_{j} \leqq \sum_{i<m} a_{i}
$$

and this contradicts (i).

Our immediate task is to obtain a kind of converse of 1.7 , that is, to show that every partial measure on a subset of $A$ can be extended to any larger subset of $A$. We start with the following definition.

Definition 1.11. Let $f$ be a partial measure on a subset $S$ of a Boolean algebra $A$, and let $x \in A$. We define $f_{0}(x)$ (the exterior measure of $x$ with respect to $f$ ) as the greatest lower bound of numbers $\xi$ of the form

$$
\xi=\left[\sum_{i<n} f\left(a_{i}\right)-\sum_{j<p} f\left(b_{i}\right)\right] / m,
$$

where $a_{i}, b_{j} \in S$ for $i<n, j<p$, and where

$$
\left\langle b_{0}, \cdots, b_{p-1}, x_{0}, \cdots, x_{m-1}\right\rangle \leqq\left\langle a_{0}, \cdots, a_{n-1}\right\rangle
$$


provided $x_{j}=x$ for every $j<m$.

Similarly $f_{i}(x)$ (the interior measure of $x$ with respect to $f$ ) is the least upper bound of numbers $\xi$ of the form (i), where $a_{i}, b_{j} \in S$ for $i<n, j<p$, and where

$$
\left\langle a_{0}, \cdots, a_{n-1}\right\rangle \leqq\left\langle b_{0}, \cdots, b_{p-1}, x_{0}, \cdots, x_{m-1}\right\rangle
$$

provided $x_{j}=x$ for every $j<m$.

It may be noticed that 1.11 cannot be simplified by taking in it $m=1$; for the values of $f_{e}(x)$ and $f_{i}(x)$ would in general change, and Theorem 1.19 below would no longer be true. When applied to a subalgebra $S$ of $A, 1.11$ agrees with the definitions of exterior and interior measures commonly used in analogous situations. In fact, we have the following theorem.

Theorem 1.12. If $f$ is a (partial) measure on a subalgebra $B$ of $A$, then $f_{i}(x)$ is the least upper bound of all numbers $f(y)$ with $y \leqq x, y \in B$, and $f_{0}(x)$ is the greatest lower bound of all numbers $f(y)$ with $y \geqq x, y \in B$.

Proof. Let $g(x)$ be the least upper bound of all numbers $f(y)$ with $y \leqq x$ $y \in B$. Then, by $1.11, f_{i}(x) \geqq g(x)$. Let $\epsilon$ be an arbitrary positive number and choose a pair of sequences $\left\langle a_{0}, \cdots, a_{k-1}\right\rangle,\left\langle b_{0}, \cdots, b_{k-1}\right\rangle$ of elements in $B$ such that

$$
\left\langle a_{0}, \cdots, a_{k-1}\right\rangle \leqq\left\langle b_{0}, \cdots, b_{k-1}, x_{0}, \cdots, x_{m-1}\right\rangle,
$$

where $x_{j}=x$ for $j<m$, and

$$
m \cdot\left[f_{i}(x)-\epsilon\right]<\sum_{i<k} f\left(a_{i}\right)-\sum_{i<k} f\left(b_{i}\right) .
$$

Note that $1.5(\mathrm{v})$ and 1.8 (i) allow us to assume that the two sequences have the same number $k$ of terms. By 1.5 (vii), 1.7, and 1.3, we may assume $a_{i} \geqq a_{i+1}$ and $b_{i} \geqq b_{i+1}$ for $i<k-1$. Let $c_{i}=a_{i} \cdot \overline{b_{i}}$ and $d_{i}=b_{i} \cdot \overline{a_{i}}$ for $i<k$. Then

$$
\left\langle c_{0}, \cdots, c_{k-1}\right\rangle \leqq\left\langle d_{0}, \cdots, d_{k-1}, x_{0}, \cdots, x_{m-1}\right\rangle
$$

by 1.5 (vii) and 1.5 (iv), and

$$
f\left(c_{i}\right)-f\left(d_{i}\right)=f\left(a_{i}\right)-f\left(b_{i}\right) \text { for } i<k
$$

by 1.3 . Since each element $d_{i}$ is disjoint with all the elements $c_{0}, \cdots, c_{k-1}$, we have

$$
\left\langle c_{0}, \cdots, c_{k-1}\right\rangle \leqq\left\langle x_{0}, \cdots, x_{m-1}\right\rangle
$$

by 1.5 (vi). Let

$$
e_{i}=\sum_{r \in s^{k, i}} \prod_{j \leqq i} c_{r_{j}}
$$

whenever $i$ is less than the larger of $k$ and $m$ ( $S^{k, i}$ having the meaning indicated in 1.3). Then, by 1.5 (vii), $e_{i} \leqq x$ for $i<m$ and, by $1.4, e_{i}=0$ for $i \geqq m$. Hence 


$$
m \cdot g(x) \geqq \sum_{i<m} f\left(e_{i}\right)=\sum_{i<k} f\left(e_{i}\right),
$$

and

$$
\begin{aligned}
m \cdot g(x) & \geqq \sum_{i<k} f\left(e_{i}\right)=\sum_{i<k} f\left(c_{i}\right) \geqq \sum_{i<k} f\left(c_{i}\right)-\sum_{i<k} f\left(d_{i}\right) \\
& =\sum_{i<k} f\left(a_{i}\right)-\sum_{i<k} f\left(b_{i}\right)>m \cdot\left[f_{i}(x)-\epsilon\right] .
\end{aligned}
$$

By the arbitrary nature of $\epsilon$, we have $g(x) \geqq f_{i}(x)$, and hence the conclusion $g(x)=f_{i}(x)$. The proof for $f_{e}(x)$ is analogous.

THEOREM 1.13. If $f$ is a partial measure on $S \subseteq A$, and $x \in A$, then $0 \leqq f_{i}(x)$ $\leqq f_{e}(x) \leqq 1$.

Proof. The inequalities $0 \leqq f_{i}(x)$ and $f_{0}(x) \leqq 1$ are immediate consequences of 1.11 since $\langle 0\rangle \leqq\langle x\rangle \leqq\langle 1\rangle$. If

$$
\left\langle a_{0}, \cdots, a_{p-1}\right\rangle \leqq\left\langle b_{0}, \cdots, b_{q-1}, x_{0}, \cdots, x_{m-1}\right\rangle
$$

and

$$
\left\langle c_{0}, \cdots, c_{r-1}, y_{0}, \cdots, y_{n-1}\right\rangle \leqq\left\langle d_{0}, \cdots, d_{s-1}\right\rangle
$$

where $x_{i}=x$ for $i<m$ and $y_{j}=x$ for $j<n$, then by 1.5 (iii) and 1.5 (i)

$$
\left\langle a_{0}, \cdots, a_{p-1}, \cdots\right\rangle \leqq\left\langle b_{0}, \cdots, b_{q-1}, \cdots, x_{0}, \cdots, x_{m-1}, \cdots\right\rangle,
$$

each of the terms $a_{0}, \cdots, a_{p-1}, b_{0}, \cdots, b_{q-1}, x_{0}, \cdots, x_{m-1}$ occurring $n$ times, and

$$
\left\langle c_{0}, \cdots, c_{r-1}, \cdots, y_{0}, \cdots, y_{n-1}, \cdots\right\rangle \leqq\left\langle d_{0}, \cdots, d_{q-1}, \cdots\right\rangle,
$$

each term occurring $m$ times. Hence, by 1.5 (iii) and 1.5 (iv),

$$
\begin{aligned}
\left\langle a_{0}, \cdots, a_{p-1}, \cdots, c_{0}, \cdots, c_{r-1},\right. & \cdots\rangle \\
& \leqq\left\langle b_{0}, \cdots, b_{q-1}, \cdots, d_{0}, \cdots, d_{s-1}, \cdots\right\rangle,
\end{aligned}
$$

the $a_{i}$ and $b_{i}$ being repeated $n$ times, and the $c_{i}$ and $d_{i}$ being repeated $m$ times. Therefore, by 1.6 ,

$$
\left[\sum_{i<p} f\left(a_{i}\right)-\sum_{i<q} f\left(b_{i}\right)\right] / m \leqq\left[\sum_{i<s} f\left(d_{i}\right)-\sum_{i<r} f\left(c_{i}\right)\right] / n .
$$

The inequality $f_{i}(x) \leqq f_{0}(x)$ is now obvious by 1.11 .

THEOREM 1.14. If $f$ is a partial measure on $S \subseteq A$, and $x \in S$, then $f_{i}(x)$ $=f_{0}(x)=f(x)$.

Proof. We need only notice that $\langle x\rangle \leqq\langle x\rangle$ and apply 1.11 .

Using the proof of 1.13 as a model it will be easy to adapt standard meth- 
ods of measure theory to give a proof of the following theorem.

ThEOREM 1.15. If $f$ is a partial measure on $S \subseteq A$, and if $x, y \in A$ and $x \cdot y$ $=0$, then

$$
f_{i}(x)+f_{i}(y) \leqq f_{i}(x+y) \leqq f_{i}(x)+f_{e}(y) \leqq f_{e}(x+y) \leqq f_{e}(x)+f_{e}(y) .
$$

As immediate consequences of 1.14 and 1.15 we obtain:

CoRollary 1.16. If $f$ is a partial measure on $S \subseteq A$, and if $x \in S, y \in A$, and $x \cdot y=0$, then

$$
f_{i}(x+y)=f(x)+f_{i}(y) \text { and } f_{e}(x+y)=f(x)+f_{e}(y) .
$$

Corollary 1.17. If $f$ is a partial measure on $S \subseteq A$, and if $x, y \in A$, $x+y \in S$, and $x \cdot y=0$, then

$$
f(x+y)=f_{i}(x)+f_{e}(y) .
$$

CoRollary 1.18. If $f$ is a partial measure on $S \subseteq A$, and $x \in A$, then

$$
f_{i}(x)+f_{e}(\bar{x})=f_{e}(x)+f_{i}(\bar{x})=1 .
$$

THEOREM 1.19. If $f$ is a partial measure on $S \subseteq A, x \in A$, and $g$ is a function on $S \cup\{x\}$ which agrees with $f$ on $S$, then $g$ is a partial measure on $S \cup\{x\}$ if and only if $f_{i}(x) \leqq g(x) \leqq f_{e}(x)$.

Proof. Suppose $f_{i}(x) \leqq g(x) \leqq f_{e}(x)$, and suppose

$$
\left\langle a_{0}, \cdots, a_{k-1}, x_{0}, \cdots, x_{m-1}\right\rangle \leqq\left\langle b_{0}, \cdots, b_{p-1}, y_{0}, \cdots, y_{n-1}\right\rangle
$$

where $a_{i} \in S, b_{j} \in S$ for $i<k, j<p$, and $x_{i}=x, y_{j}=x$ for $i<m, j<n$. We must show

$$
m \cdot g(x)+\sum_{i<k} g\left(a_{i}\right) \leqq n \cdot g(x)+\sum_{j<p} g\left(b_{j}\right) .
$$

This being an immediate consequence of 1.5 (iv) when $m=n$, assume $n-m$ $=r>0$. By 1.5 (iv),

$$
\left\langle a_{0}, \cdots, a_{k-1}\right\rangle \leqq\left\langle b_{0}, \cdots, b_{p-1}, y_{0}, \cdots, y_{r-1}\right\rangle .
$$

Hence

$$
r \cdot g(x) \geqq r \cdot f_{i}(x) \geqq \sum_{i<k} g\left(a_{i}\right)-\sum_{j<p} g\left(b_{j}\right),
$$

and the conclusion follows. If $n<m$, we use the fact that $g(x) \leqq f_{e}(x)$.

Conversely, suppose $g$ is a partial measure. If $\epsilon$ is a positive number, we can find elements $a_{i}, b_{j}$ of $S$ such that

$$
\left\langle a_{0}, \cdots, a_{k-1}, x_{0}, \cdots, x_{m-1}\right\rangle \leqq\left\langle b_{0}, \cdots, b_{p-1}\right\rangle
$$

with $x_{i}=x$ for $i<m$ and 


$$
m \cdot\left[f_{e}(x)+\epsilon\right]<\sum_{j<p} g\left(b_{j}\right)-\sum_{i<k} g\left(a_{i}\right)
$$

But

$$
m \cdot g(x)+\sum_{i<k} g\left(a_{i}\right) \leqq \sum_{j<p} g\left(b_{j}\right) .
$$

Hence $g(x)<f_{0}(x)+\epsilon$ for arbitrary $\epsilon>0$, and therefore $g(x) \leqq f_{0}(x)$. Similarly $g(x) \geqq f_{i}(x)$, and the proof is complete.

THEOREM 1.20. If $f$ is a partial measure on $S \subseteq A$ which assumes only the values 0 and 1 , then $f_{i}$ and $f_{e}$ assume only the values 0 and 1 on the whole algebra A.

Proof. Let $x \in A$, and let $S_{0}$ be the set of elements $y \in S$ for which $f(y)=0$. We put $S_{1}=S-S_{0}$. If, for some choice of elements $a_{i} \in S_{0}, b_{j} \in S_{1}$, we have

$$
\bar{x}+\sum_{j<p} \overline{b_{j}}+\sum_{i<n} a_{i}=1,
$$

then

$$
x \leqq \sum_{i<n} a_{i}+\sum_{j<p} \overline{b_{j}},
$$

and, by 1.18 and $1.15, f_{e}(x)=0$. If, on the other hand, (1) always fails, then by 1.10 the function $g$ on $S \cup\{x\}$ which agrees with $f$ on $S$ and for which $g(x)=1$ is a partial measure on $S \cup\{x\}$. Hence, by $1.19, f_{e}(x) \geqq g(x)=1$; and, by $1.13, f_{e}(x)=1$. The statement for $f_{i}$ follows from 1.18 .

THEOREM 1.21. If $f$ is a partial measure on $S$, and $S \subseteq T \subseteq A$, then there is a partial measure $g$ on $T$ which agrees with $f$ on $S$. If, moreover, $f$ assumes only the values 0 and 1, the same may be postulated for $g$.

Proof. This follows from 1.19, 1.20, and the well-ordering principle.

THEOREM 1.22. If $f$ is a partial measure on a subset $S$ of a Boolean algebra $A$ (or a measure on a subalgebra $S$ of $A$ ), there is a measure $g$ on $A$ which agrees with $f$ on $S$. If $f$ assumes only the values 0 and 1 , the same may be postulated for $g$.

Proof. This is proved by 1.21 and 1.9 (ii).

CoRollary 1.23. There is a measure on every Boolean algebra $A$; if $a \neq 0$ is an element of $A$, then there is a two-valued measure $g$ on $A$ with $g(a)=1$.

Proof. The function $g$ on $\{a\} \cup\{1\}$ with $g(a)=g(1)=1$ is a partial measure by 1.10 , and hence we may apply to it 1.22 .

According to 1.9 (ii), 1.7, and 1.22, Definition 1.6 characterizes adequately and intrinsically the behavior of a measure restricted to an arbitrary subset $S$ (containing 1 ) of its original domain.

We wish to point out here some consequences of our discussion which are 
less closely related to the main ideas of this paper.

From 1.21 we easily see that, if $f$ is a partial measure on $S \subseteq T \subseteq A$, and $x \in T$, then the set of values of $g(x)$ as $g$ ranges over all partial measures on $T$ which agree with $f$ on $S$ is the closed interval with the end points $f_{i}(x)$ and $f_{e}(x)$. This result (especially when applied to the case $T=A$ ) gives the possibility of characterizing the inner and outer measures $f_{i}$ and $f_{0}$ in a way different essentially from that used in 1.11 .

Given a partial measure $f$ on a subset $S$ of $A$, an element $x$ in $A$ may be called measurable with respect to $f$ if $f_{i}(x)=f_{e}(x)$. In view of 1.19 and 1.21 we can also say that $x$ is measurable if $f$ can be extended from $S$ to $S \cup\{x\}$ in exactly one way, or if every measure on $A$ which agrees with $f$ on $S$ agrees with $f$ also on $\{x\}$. In view of 1.8 (i) the element 0 is measurable. By 1.14 every element $x$ in $S$ is measurable; in particular, 1 is measurable. From 1.15 it follows that, $x, y, u$, and $v$ being any four elements in $A$ with $x+y=u$ and $x \cdot y=v$, if three of them are measurable, then the fourth also is measurable; in particular, if an element $x$ is measurable, the same holds for $\bar{x}$. Hence we can conclude, for instance, that if the set $S$ is closed under addition or under multiplication, then every element in the subalgebra $T$ generated by $S$ is measurable; thus in this case there exists only one measure $g$ on $T$ which agrees with $f$ on $S$.

As the last theorem in this section we give a result of a more special nature which will find an application in $\$ 2$.

THEOREM 1.24. For every Boolean algebra $A$ the following three conditions are equivalent:

(i) $A$ is isomorphic with a field of subsets of the set of natural numbers.

(ii) $A$ is a subalgebra of a separable Boolean algebra.

(iii) There exists an infinite sequence of measures $f_{0}, f_{1}, \cdots$ on $A$ such that, for every element $x \neq 0$ in $A$, we have $f_{n}(x)=1$ for some measure $f_{n}$ of the sequence.

The measures $f_{0}, f_{1}, \cdots$ in (iii) may be assumed to be two-valued.

Proof. From the fact that $A$ is isomorphic to a subalgebra of a separable algebra we conclude in a familiar way that $A$ itself can be imbedded as a subalgebra in a separable algebra; hence (i) implies (ii).

To derive (iii) from (ii) we assume that $A$ is a subalgebra of a separable Boolean algebra $A^{\prime}$. Let $d_{0}, d_{1}, \cdots$ be elements (different from 0 ) which constitute a set dense in $A^{\prime}$. By 1.23 there are measures $f_{0}^{\prime}, f_{1}^{\prime}, \cdots$ on $A^{\prime}$ such that $f_{n}^{\prime}\left(d_{n}\right)=1$ for $n=0,1,2, \ldots$. In view of 1.1 (iii) and 1.2 (ii), for any given element $x \neq 0$ in $A^{\prime}$, there is a natural number $n$ such that $f_{n}^{\prime}(x)=1$. Let now $f_{n}$ be the function on the set $A$ which agrees with $f_{n}^{\prime}$ on this set $(n=0,1,2, \cdots)$. By 1.7 and 1.9 (ii), the functions $f_{0}, f_{1}, \cdots$ just defined satisfy condition (iii) of our theorem.

Assume now that (iii) holds. Let $f_{0}, f_{1}, \cdots$ be measures satisfying condition (iii). We first show that these measures can be replaced by two-valued 
measures $h_{0}, h_{1}, \cdots$ satisfying the same condition. In fact, for $n=0,1$, $2, \cdots$, let $S_{n}$ be the set of all $x \in A$ such that $f_{n}(x)=1$, and let $g_{n}$ be the function obtained from $f_{n}$ by restricting its domain to $S_{n}$. By 1.7 and 1.9 (ii), $g_{n}$ is a partial measure on $S_{n}$, which clearly assumes 1 as the only value. By 1.22 there is a two-valued measure $h_{n}$ on $A$ which agrees with $g_{n}$, and hence also with $f_{n}$, on $S_{n}$. The measures $h_{0}, h_{1}, \cdots$ thus obtained obviously satisfy (iii). Now for every $x \in A$ let $H(x)$ be the set of all natural numbers $n$ such that $h_{n}(x)=1$. A familiar argument shows that the family of all sets $H(x)$ of natural numbers just defined is a field of sets (with the set of all natural numbers as an element) and that the function $H$ maps the algebra $A$ isomorphically onto this field $\left({ }^{3}\right)$. Thus (iii) implies (i); and the proof is complete.

The fact that 1.24 (ii) implies 1.24 (i) can be expressed in the following way: the Boolean algebra of all sets of natural numbers is a universal algebra for all separable Boolean algebras and for all subalgebras of these algebras. It may be noticed in this connection that a subalgebra of a separable Boolean algebra is not necessarily separable itself. For instance, the separable Boolean algebra of all sets of natural numbers has a free subalgebra with uncountably many generators $\left({ }^{4}\right)$, and the latter is easily seen not to be separable.

In the proof of 1.24 we have made an essential use of Theorem 1.22 and Corollary 1.23 which were established by transfinite methods. We do not know any constructive proof of Theorem 1.24 even in its application to certain special Boolean algebras. For instance, the algebra of all Borel sets of Euclidean space divided by the ideal of Borel sets of the first category is known to be separable $\left(^{5}\right)$ and hence to satisfy 1.24 (ii), but we do not know how to prove without using transfinite methods that this algebra also satisfies the remaining conditions of 1.24 . It may be noticed, however, that the proofs of $1.22,1.23$, and hence also 1.24 , can be carried through in a constructive way if we restrict ourselves to countable Boolean algebras. Analogous remarks apply to two other theorems in our further discussion which involve the notion of a separable Boolean algebra, in fact, to 2.5 and 3.10.

There are certain familiar relations between the notion of a measure and that of an ideal $\left.{ }^{6}\right)$. If $f$ is a measure, then the set $I$ of all $x$ with $f(x)=0$ is an ideal different from the whole algebra $A$; if in particular $f$ is a two-valued measure, the set $I$ is a prime ideal. Conversely, if $I$ is an ideal $\neq A$ and if we define a function $f$ on $I \cup\{1\}$ with $f(x)=0$ for $x$ in $I$ and $f(1)=1$, then by 1.10 , $f$ is a partial measure on $I \cup\{1\}$; and if $I$ is a prime ideal, then the function $g$ with $g(x)=0$ for $x$ in $I$ and $g(x)=1$ for $x$ not in $I$ is a two-valued measure

(3) The argument is essentially the same as that used in proving the general representation theorem for Boolean algebras in Stone [3, chap. IV].

(4) This follows directly from Lemma 3.16 in Tarski [3, part I, p. 61] (where further references can also be found).

(5) See Birkhoff $[1$, p. 103], where further references to J. von Neumann and S. Ulam are given.

(6) Cf. Tarski [3, part II, pp. 55 ff.]. 
on $A$. Hence the second part of 1.22 is simply another formulation of the well known theorem which states that every ideal in a Boolean algebra $A$ which is different from $A$ is included in a prime ideal. Similarly, condition 1.24 (iii) can be reformulated as follows: there exists in $A$ an infinite sequence of ideals $I_{0}, I_{1}, \ldots$ which are different from $A$ such that every element $x \neq 1$ in $A$ belongs to at least one of them; $I_{0}, I_{1}, \ldots$ can be assumed to be prime ideals.

2. Strictly positive measure.

Definition 2.1. A measure $f$ on a Boolean algebra $A$ is called strictly positive if $f(x)=0$ only for $x=0$.

From a certain point of view strictly positive measure and two-valued measure are two extreme cases in the variety of all posible measures. A measure $f$ is strictly positive if it vanishes for only one element of the algebra; a measure is two-valued if it vanishes on as large a set as any measure can vanish.

The problem under which conditions a Boolean algebra $A$ has a strictly positive measure is essentially equivalent to the following (apparently much more general) problem: given an algebra $A$ and a set $I \subseteq A$, under which conditions does there exist a measure on $A$ which vanishes for all elements $x$ in $I$ and for no other elements? In fact, it is easily seen that for the existence of such a measure it is necessary and sufficient that $I$ be an ideal in $A$ and that the quotient algebra $A / I$ have a strictly positive measure.

No workable criteria (conditions which are both necessary and sufficient) for the existence of a strictly positive measure in an arbitrary Boolean algebra are known. We are going to establish here a few necessary conditions (Theorem 2.4), some of which may prove to be sufficient, and also a sufficient condition (Theorem 2.5), which however is far from being necessary. To formulate one of the necessary conditions conveniently, we introduce the notion of a ramification $\operatorname{set}\left({ }^{7}\right)$ :

Definition 2.2. $A$ set $R$ of elements of a Boolean algebra $A$ is called a ramification set if (i) for any elements $x$ and $y$ in $R$, either $x \cdot y=0$ or $x \geqq y$ or $x \leqq y$, and (ii) for every element $x$ in $R$, the set of all elements $y$ in $R$ with $x \leqq y$ is well ordered by the relation $\geqq$.

For later reference we give the following theorem.

THEOREM 2.3. Every set $S$ of elements in a Boolean algebra includes a ramification set $R$ with the following property: for every element $x \in S-R$ there is an element $y \in R$ such that $x \cdot y \neq 0$ and $x \cdot \bar{y} \neq 0$.

Proof. There are certainly ramification sets included in $S$, for example, the empty set. If $R_{1}$ and $R_{2}$ are ramification sets $\subseteq S$, we agree to say that $R_{1} \prec R_{2}$ if $R_{1} \subseteq R_{2}$ and if, whenever $x \in R_{1}$ and $y \in R_{2}-R_{1}$, we have $x \cdot y=0$ or $x>y$. The relation $\prec$ thus defined establishes a partial ordering in the family

(7) Compare Kurepa [1, pp. 72 ff.]. 
of all ramification sets $\subseteq S$. From 2.2 it is seen that the union of any family of ramification sets $\subseteq S$ which is well ordered by $\prec$ is again a ramification set $\subseteq S$. Hence, by the well-ordering principle, there is a ramification set $R \subseteq S$ which is maximal in the sense that for no ramification set $R^{\prime} \subseteq S$ which is different from $R$ do we have $R<R^{\prime}$. Consequently, if $x \in S-R$, there must be an element $y \in R$ such that $x \cdot y \neq 0$ and $x \cdot \bar{y} \neq 0$; for otherwise $R^{\prime}=R \cup\{x\}$ would be a ramification set $\subseteq S$ with $R<R^{\prime}$. This completes the proof.

TheOREM 2.4. For an arbitrary Boolean algebra $A$, each of the following conditions implies all the succeeding ones:

(i) There is a strictly positive measure on $A$.

(ii) $A$ can be represented as the union of an infinite sequence of sets $S_{0}$, $S_{1}, \cdots$ none of which includes an infinite subset of pairwise disjoint elements.

(iii) Every uncountable subset $S$ of $A$ includes an uncountable subset in which no two elements are disjoint.

(iv) Every ramification set in $A$ is countable.

(v) Every set of pairwise disjoint elements of $A$ is countable.

Proof. We start with the implication (i) $\rightarrow$ (ii). If $f$ is a strictly positive measure on $A$, let $S_{0}=\{0\}$ and let $S_{n}$, for $n>0$, be the set of elements $x$ for which $f(x) \geqq 1 / n$. Clearly the sets $S_{0}, S_{1}, \ldots$ satisfy (ii).

(ii) $\rightarrow$ (iii): It is known that, in general (that is, independent of assumption (ii)), every uncountable set $T$ of elements of a Boolean algebra either includes an infinite subset of pairwise disjoint elements or else includes an uncountable subset no two elements of which are disjoint $\left({ }^{8}\right)$. Briefly this can be shown as follows. Either every uncountable subset $V$ of $T$ has an element $x$ disjoint with uncountably many elements of $V$, or else there is an uncountable subset $W$ of $T$ such that every element of $W$ is disjoint with at most countably many elements of $W$. If the first case holds, it is easy to construct an infinite set of pairwise disjoint elements of $T$. In the second case, by well ordering $W$, we can easily find an uncountable subset of $W$ in which no two elements are disjoint.

Now let $S$ be any uncountable subset of $A$, and let $S_{0}, S_{1}, \ldots$ be the sequence in (ii). For some $n, S \cap S_{n}$ is uncountable and includes no infinite subset of pairwise disjoint elements. Hence, by what has just been shown, the set $S \cap S_{n}$, and therefore also $S$ itself, includes an uncountable subset without disjoint elements.

(iii) $\rightarrow$ (iv): We first notice that (iii) obviously implies (v). Suppose now that there is an uncountable ramification set $R$ in $A$. According to (iii) there exists an uncountable subset $T$ of $R$ no two elements of which are disjoint. From 2.2 it is easily seen that $T$ is well ordered by $\geqq$. Consider the set $V$ of all elements $v$ of the form $v=x \cdot \bar{y}$, where $x$ and $y$ are elements of $T$ such that $x>y$ and that $x>z>y$ holds for no $z$ in $T$. Clearly $V$ is an uncountable set of

(8) This is a consequence of a more general result in Dushnik-Miller [1]. 
pairwise disjoint elements, which contradicts ( $v$ ) and hence also (iii).

(iv) $\rightarrow$ (v): It suffices to notice that every set of pairwise disjoint elements is a ramification set.

It is not known whether any two of the conditions (i)-(v) of Theorem 2.4 are equivalent for arbitrary Boolean algebras. Hence the problem whether any one of the conditions (ii)-(v) is sufficient for the existence of a strictly positive measure also remains open $\left({ }^{9}\right)$. (Regarding the equivalence of (iv) and (v), see the last theorem in this section.)

We wish to mention here several conditions which are closely related to conditions (i) $-(\mathrm{v})$ of $2.4\left({ }^{10}\right)$.

(ii') The algebra $A$ can be represented as the union of an infinite sequence of sets $S_{0}, S_{1}, \cdots$ such that for each $n$ every set of pairwise disjoint elements of $S_{n}$ has at most $n$ members.

Condition (ii') is implied by (i) (as is seen from the proof of (i) $\rightarrow$ (ii)) and obviously implies (ii). We do not know whether (ii') is equivalent to (i) or (ii).

(iii') Every uncountable subset $S$ of $A$ includes an uncountable subset $T$ such that every set of pairwise disjoint elements of $T$ is finite.

Obviously, (iii) implies (iii'), and it is easily seen from the proof of (ii) $\rightarrow$ (iii) that the implication in the opposite direction also holds.

$\left(v^{\prime}\right)$ Every uncountable subset $S$ of $A$ includes an uncountable subset $T$ such that every set of pairwise disjoint elements of $T$ is countable.

$\left(v^{\prime \prime}\right)$ Every uncountable subset $S$ of $A$ includes an infinite subset $T$ with no disjoint elements.

$\left(v^{\prime \prime \prime}\right)$ Every uncountable subset $S$ of $A$ contains an element $x$ such that the set of all elements of $S$ which are not disjoint with $x$ is uncountable.

Each of the conditions $\left(v^{\prime}\right),\left(v^{\prime \prime}\right)$, and $\left(v^{\prime \prime \prime}\right)$ obviously implies $(v)$. From (v) we immediately derive $\left(\mathrm{v}^{\prime}\right)$ by contradiction. To derive $\left(\mathrm{v}^{\prime \prime}\right)$ and $\left(\mathrm{v}^{\prime \prime \prime}\right)$ from (v) we apply arguments analogous to those used in the proof of (ii) $\rightarrow$ (iii). In particular, in deriving $\left(v^{\prime \prime}\right)$, we use the fact that generally every uncountable set $S$ of elements of an arbitrary Boolean algebra includes either an uncountable set of pairwise disjoint elements or an infinite set of elements no two of which are disjoint $\left({ }^{8}\right)$. We can obtain some further conditions equivalent to $(\mathrm{v})$ by replacing in $\left(\mathrm{v}^{\prime \prime}\right)$ the words "with no disjoint elements" by

(9) The problem whether condition 2.4 (v) is sufficient for the existence of a strictly positive measure was formulated (and the rather obvious proof of the necessity of this condition was given) in Tarski [3, part II, pp. $55 \mathrm{ff}$.]. Condition 2.4 (iii) was discussed in Knaster [1] but not in connection with the measure problem; the problem whether 2.4 (iii) is necessary and sufficient for the existence of a strictly positive measure, and to which our Theorem 2.4 gives a partial answer, was communicated to us by E. Szpilrajn-Marczewski. It may be mentioned that an example of a family of sets (but not of a field of sets, and hence not of a Boolean algebra) which satisfies 2.4 (v) but not 2.4 (iii) has been found; see Knaster [1] and Szpilrajn-Marczewski [2], where a construction in Sierpinski [2] has been used.

(10) For some further related conditions cf. Knaster [1]. 
"such that every set of pairwise disjoint elements of $T$ is finite," or else by changing in $\left(\mathrm{v}^{\prime \prime}\right)$ the last word "uncountable" to "infinite."

(vi) Every subset of $A$ which is well ordered by $\geqq$ is countable.

From the proof of (iii) $\rightarrow$ (iv) we see that (v) implies (vi). In general (vi) does not imply (v); for instance, the Boolean algebra constituted by all finite subsets of an uncountable set $U$ and by their complements with respect to $U$ clearly satisfies (vi) but not (v). If, however, the Boolean algebra $A$ is countably complete, conditions (v) and (vi) are equivalent.

As an application of 2.4 consider the Boolean algebra $A$ of all sets of natural numbers and the family $I$ of all finite sets of natural numbers. $I$ is clearly an ideal in $A$, and it is known that the quotient algebra $A / I$ does not satisfy condition (v) of 2.4 , nor even condition (vi)(11). Hence there is no strictly positive measure on $A / I$; or, in other words, there is no measure on all sets of natural numbers which vanishes on those and only those sets which are finite.

ThEOREM 2.5. There is a strictly positive measure on every separable Boolean algebra $A$.

Proof. Let $\alpha_{0}, \alpha_{1}, \cdots$ be any positive numbers with sum 1. By putting for every set $X$ of natural numbers

$$
f(X)=\sum_{n \in X} \alpha_{n}
$$

we clearly obtain a strictly positive measure $f$ on the algebra of all sets of natural numbers. Hence our theorem follows immediately by 1.24.

Theorem 2.5 can also be derived, independently of 1.24 , from certain results which can be found in the literature. The proof essentially reduces to the case when the algebra $A$ is atomless; and in treating this case we use the following facts:

I. Every separable atomless Boolean algebra can be imbedded as a subalgebra in a complete separable atomless Boolean algebra.

II. Any two complete separable atomless Boolean algebras are isomorphic.

III. The Boolean algebra of Borel sets of a bounded interval divided by the ideal of Borel sets of the first category is a complete separable atomless Boolean algebra.

IV. The Boolean algebra in III has a strictly positive measure $\left({ }^{12}\right)$.

(ii) See Sierpiński [3].

(12) For Statement I, compare Stone [1, p. 236] and Tarski [4, pp. 177 ff.], where further references, in particular to $H$. M. MacNeille, can be found. Statement II is a result of $S$. Jaskowski; see Tarski [4, p. 179]. The proof of II is exactly analogous to the proofs of well known set-theoretical theorems which characterize the order of the set of rational numbers and the set of real numbers; compare, for example, Sierpinski [1, pp. $145 \mathrm{ff}$.$] . Regarding$ Statement III, see footnote 5. For Statement IV, see Tarski [5, p. 229] (and, in particular, footnote 6 on that page referring to E. Szpilrajn-Marczewski). 
While conditions (ii)-(v) of 2.4 are not known to be sufficient for a Boolean algebra to have a strictly positive measure, the condition of separability in 2.5 is certainly not necessary. The Boolean algebra of all sets measurable in the sense of Lebesgue divided by the ideal of all sets of measure 0 can serve as a counter-example $\left({ }^{13}\right)$; the same applies to an arbitrary free Boolean algebra with uncountably many generators. We are now going to consider a rather special class of Boolean algebras for which all the conditions just mentioned except 2.4 (v) prove to be equivalent (see 2.9 below).

Definition 2.6. A Boolean algebra $A$ is said to be an algebra with an ordered basis $B$ if $B$ is a subset of $A$ which is simply ordered by the relation $\leqq$ and which generates $A\left({ }^{14}\right)$.

THEOREM 2.7. Let $A$ be an algebra with an ordered basis $B$. We then have:

(i) The set $B^{\prime}=B \cup\{0\} \cup\{1\}$ is also an ordered basis of $A$.

(ii) If 0 and 1 are in $B$, every element $a \in A$ has a unique representation of the form

$$
a=\sum_{i<n} b_{2 i+1} \cdot \overline{b_{2 i}}
$$

where $b_{0}, \cdots, b_{2 n-1}$ are in $B$, and $b_{j}<b_{j+1}$ for $j<2 n-1$.

Proof. The proof of (i) is obvious, and the proof of (ii) is essentially known.

It can be shown that all countable Boolean algebras are algebras with an ordered basis $\left({ }^{15}\right)$. The following representation theorem provides us with further examples of this class of algebras.

THEOREM 2.8. (i) The interval algebra of a simply ordered set (and hence every isomorphic algebra) is a Boolean algebra with an ordered basis.

(ii) Conversely, every Boolean algebra with an ordered basis is isomorphic with the interval algebra of a simply ordered set.

Proof. (i) If $S$ is a set which is simply ordered by a relation $\prec$, then all intervals of the form

$$
\underset{x}{\mathrm{E}}[x \in S, x<b]
$$

where $b$ is an arbitrary element of $S$, clearly form an ordered basis of the interval algebra of $S$.

(ii) Let $A$ be a Boolean algebra with an ordered basis $B$. By 2.7 (i) we may assume 0 and 1 are in $B$. Let $B^{\prime}=B-\{0\}$. Clearly, $B^{\prime}$ is still simply

(13) Cf. Birkhoff [1, p. 103], where further references are given.

(14) For this definition and for Theorems 2.7 and 2.8 (i), see Mostowski-Tarski [1].

(15) This result can easily be obtained by using a theorem of Stone [2, p. 393]. (The result was included in a joint paper of A. Mostowski and A. Tarski which was to appear in Fundamenta Mathematicae, vol. 33, but whose manuscript was destroyed during the last war.) 
ordered by the (Boolean algebraic) relation $\leqq$. By 2.7 (ii) every element $a$ in $A$ has a unique representation

$$
a=\sum_{i<n} b_{2 i+1} \cdot \overline{b_{2 i}}
$$

where $b_{j} \in B$ for $j<2 n$ and $b_{0}<\cdots<b_{2 n-1}$. We correlate with $a$ the sum $f(a)$ of all intervals

$$
\underset{x}{\mathrm{E}}\left[x \in B, b_{2 i}<x \leqq b_{2 i+1}\right], \quad i=0, \cdots, n-1 .
$$

As is easily seen, the function $f$ maps $A$ isomorphically onto the interval algebra of $B^{\prime}$ (and not of $B$, since 0 does not belong to any interval sum $f(a)$; $f(0)$ is the empty interval).

Returning to the measure problem, we obtain the following theorem.

THEOREM 2.9. If $A$ is a Boolean algebra with an ordered basis, then conditions (i)-(iv) of 2.4 are equivalent to each other and also to the condition

(o) $A$ is separable.

Proof. In view of 2.4 and 2.5 we have only to show that condition 2.4 (iv) implies condition (o). By hypothesis, $A$ has an ordered basis $B$. Let $I$ be the set of all elements $b_{1} \cdot \bar{b}_{0}$ where $b_{0}$ and $b_{1}$ are in $B$ and $b_{0}<b_{1}$. Every element $i=b_{1} \cdot \overline{b_{0}}$ in $I$ may be referred to as an interval, and the elements $b_{0}, b_{1}$ as its end points; by 2.7 (ii) every interval has exactly two end points. By $2.3, I$ includes a ramification set $R$ with the following property: for every element $i \in I-R$ there is an element $r \in R$ such that neither $i \cdot r=0$ nor $i<r$ holds. Condition 2.4 (iv) implies that $R$ is countable. Hence the set $S$ of all end points of intervals in $R$ is also countable; and the same applies to the set $D$ of all intervals whose end points are in $S$. Clearly $R \subseteq D$ and 0 is not in $D$. We are going to show that $D$ is dense in $A$. In fact, consider an arbitrary element $a \neq 0$ in $A$. By 2.7 (ii) there is an interval $i_{0} \leqq a$. If $i_{0} \geqq r$ for some $r$ in $R, r$ is also in $D$, and we have found an element in $D$ which is included in $a$. Otherwise $i_{0} \in I-R$. Therefore there must be an element $r_{0}$ in $R$ such that neither $i_{0} \cdot r_{0}=0$ nor $i_{0} \leqq r_{0}$ nor $i_{0} \geqq r_{0}$. If now $b_{0}$ and $b_{1}$ are the end points of $i_{0}$, we conclude that one of the end points of $r_{0}$, say $b_{2}$, satisfies the condition

$$
b_{0}<b_{2}<b_{1} \text {. }
$$

By the definition of $S$, we have $b_{2} \in S$. By applying the same argument to the interval $i_{1}=b_{1} \cdot \overline{b_{2}}$ instead of $i_{0}$, we conclude that there is another element $b_{3} \in S$ such that

$$
b_{0}<b_{2}<b_{3}<b_{1} \text {. }
$$

We now have $i_{2}=b_{3} \cdot \overline{b_{2}} \in D$ and $i_{2}<i_{0} \leqq a$. Thus the countable set $D$ proves to be dense in $A$, and $A$ is separable. This completes the proof. 
In view of 2.8 we can reformulate 2.9 so as to make it apply directly to ordered sets. Since intervals of an ordered set $S$ are members of the interval algebra of $S$, we can apply to them all Boolean algebraic notions-in particular that of a ramification set.

THEOREM 2.10. Let $S$ be a set which is simply ordered by an arbitrary relation 〈. Then the following conditions are equivalent:

(o) There is a countable subset $D$ of $S$ which is dense in $S$.

(i) The set $S$ is similarly ordered to a set of real numbers (considered as a set simply ordered by the arithmetical $\leqq$ relation).

(ii) The set of all intervals in $S$ can be expressed as the union of an infinite sequence of sets $\mathfrak{S}_{0}, \mathfrak{S}_{1}, \cdots$ none of which includes an infinite set of pairwise disjoint intervals.

(iii) Every uncountable family of intervals in $S$ includes an uncountable subfamily in which no two intervals are disjoint.

(iv) Every ramification set of intervals of $S$ is countable. Each of these conditions implies

(v) Every set of pairwise disjoint intervals of $S$ is countable $\left.{ }^{16}\right)$.

Proof. By 2.8 (i) it suffices to show that conditions (i)-(v) of 2.4 for the interval algebra $A$ of $S$ are respectively equivalent to conditions (i)-(v) of our theorem, and that condition (o) of 2.9 is equivalent to our condition (o). The proof is easy; we outline some portions of it.

If 2.4 (i) holds for $A$, we consider a strictly positive measure $f$ on $A$. Choose an arbitrary element $a \in S$ and correlate with every element $x \in S$ a real number $g(x)$ defined as follows:

$$
\begin{array}{cc}
g(a)=0 ; & \text { for } a<x ; \\
g(x)=f(\underset{y}{\mathrm{E}}[y \in S, a<y<x, y \neq a]) & \text { for } x<a .
\end{array}
$$

The function $g$ establishes the similarity of the set $S$ and the set of real numbers which are values of $g$; thus, 2.4 (i) implies 2.10 (i).

Assume now 2.10 (iv) to hold. We have to show 2.4 (iv) holds for $A$, that is, every ramification set $R$ of finite sums of intervals is countable. By 2.8 (i) and 2.7 (ii) every finite sum $X$ of intervals has a "canonical" representation

$$
X=X_{0} \cup \cdots \cup X_{m-1},
$$

(16) A related, though less general, result can be found in Knaster [1]. Specifically it is assumed there that $S$ is a dense continuous ordered set without first and last elements, and it is shown that under these assumptions condition 2.10 (iii) is necessary and sufficient for $S$ to be similar to the set of all real numbers; no conditions analogous to 2.10 (ii) and 2.10 (iv) are involved in that discussion. 
where $X_{0}, \cdots, X_{m-1}$ are intervals such that the union of no two of them is an interval. If now $Y$ is another sum of intervals such that $Y \subseteq X$ and if

$$
Y=Y_{0} \cup \cdots \cup Y_{n-1}
$$

is its canonical representation, then, as is easily seen, each interval $Y_{j}$ is included in one of the intervals $X_{i}$. Making use of this, we replace in the ramification set $R$ each interval sum $X$ by all the intervals occurring in its canonical representation. It is clear that the set $R^{\prime}$ of intervals thus obtained is again a ramification set. Hence it is countable by 2.10 (iv), and therefore $R$ must also be countable.

Let finally 2.10 (o) be satisfied; let $D$ be a countable set dense in $S$. We consider the set $D^{\prime}$ consisting of all intervals with two end points, both in $D$, as well as of all sets $\{d\}$ with $d$ in $D$, whenever these sets are intervals in the sense accepted here. The set $D^{\prime}$ just defined is obviously countable and is easily seen to be dense in $A$ in the Boolean algebraic sense. Thus 2.10 (o) implies 2.9 (o).

The theorem just established remains valid if by an interval of an ordered set $S$ we agree to understand what is usually called a closed interval with two distinct end points; and if we use this convention in the definition of density in $S$ as well.

In connection with 2.9 the problem arises whether, for a Boolean algebra with an ordered basis, condition 2.4 (v) implies (and hence is equivalent to) those stated in 2.9. As is easily seen, this is equivalent to the problem whether, for any given simply ordered set $S$, condition 2.10 (v) implies any of the conditions (o)-(iv) of 2.10 .

The likelihood of solving this problem is at present very slight since the problem is equivalent to a famous unsolved set-theoretical problem, in fact, to the problem of Souslin $\left({ }^{17}\right)$. By the Souslin hypothesis we understand the following statement:

Every simply ordered set without jumps and gaps, without first and last elements, and in which every set of pairwise disjoint intervals is countable, is similar to the set of all real numbers.

THEOREM 2.11. The Souslin hypothesis is equivalent to each of the following statements:

(I) Every Boolean algebra which satisfies condition 2.4 (v) also satisfies 2.4 (iv).

(II) Every Boolean algebra with an ordered basis which satisfies 2.4 (v) also satifies the remaining conditions of 2.4 as well as 2.9 (o).

(III) Every ordered set which satisfies 2.10 (v) also satisfies the remaining conditions of 2.10 .

Proof. It has been shown that the Souslin hypothesis implies, and is even

(17) See Sierpinski [1, pp. 151 ff.]. 
equivalent to, the following statement:

(IV) Let $S$ be a set of elements of a Boolean algebra such that

(i) For any two elements $x, y$ in $S$, either $x \leqq y$ or $y \leqq x$ or $x \cdot y=0$.

(ii) Every subset of $S$ of pairwise disjoint elements is countable.

(iii) Every subset of $S$ no two elements of which are disjoint is countable. Then the set $S$ is itself countable $\left({ }^{18}\right)$.

To derive (I) from (IV), consider a Boolean algebra $A$ satisfying 2.4 (v) and a ramification set $R$ in $A$. It is obvious by 2.2 that the premises (i) and (ii) of (IV) are satisfied by $R$; and a simple argument shows that the same applies to (iii). (See the proof of (iii) $\rightarrow$ (iv) in 2.4.) Hence, by (IV), $R$ is countable, and (I) has been obtained.

Statement (II) follows directly from (I) by 2.9. The derivation of (III) from (II) is very easy (compare the proof of 2.10). Finally, it is almost obvious that (III) implies the Souslin hypothesis $\left({ }^{17}\right)$.

It may be noticed that, in addition to Boolean algebras with an ordered basis, there are also other classes of Boolean algebras for which conditions (i)-(iv) of 2.4 are equivalent to each other and to the condition of separability. Such is, for instance, the class of all countably distributive Boolean algebras. This follows from the fact that every countably distributive Boolean algebra which satisfies 2.4 (iv) is atomistic. A subclass of the class of countably distributive algebras is that constituted by all fields of sets which are countably complete (even in the wider sense). For this subclass, condition 2.4 (v) proves also to be equivalent to the remaining conditions of 2.4. Thus, every field of sets which is countably complete in the wider sense and has a strictly positive measure-or at least satisfies $2.4(\mathrm{v})$-is atomistic, and hence is isomorphic with a field of sets of natural numbers $\left({ }^{19}\right)$.

We have not introduced any notion which would correspond to that of a strictly positive measure but which, like that of partial measure, would apply to an arbitrary subset $S$ of elements of a Boolean algebra $A$. As a notion of this kind we could discuss that of a strictly increasing partial measure. A partial measure on $S$ is called strictly increasing if, in addition to conditions (i)-(iii) of 1.6, it satisfies the following condition:

(iv) If, under the hypothesis of 1.6 (ii),

$$
\left\langle b_{0}, \cdots, b_{n-1}\right\rangle \leqq\left\langle a_{0}, \cdots, a_{m-1}\right\rangle
$$

does not hold, then

$$
\sum_{i<m} f\left(a_{i}\right)<\sum_{j<n} f\left(b_{j}\right)
$$

We can establish for this notion an analogue of Theorem 1.9: when applied to a set $S$ which is closed under addition and multiplication condition (iv) can be replaced by the simpler one:

(18) Essentially the same result is to be found in Miller [1].

(19) The proof of these results, recently obtained by A. Tarski, will be published later. 
$\left(\mathrm{iv}^{\prime}\right)$ If $x, y$ are in $S$ and $x<y$, then $f(x)<f(y)$;

and when applied to the whole algebra or to a subalgebra of $A$, the notion of a strictly increasing partial measure coincides with that of a strictly positive measure. By restricting the domain of a strictly positive measure on a subalgebra $T$ of $A$ to a set $S \subseteq T$ (with 1 in $S$ ), we always obtain a strictly increasing partial measure on $S$. The converse does not hold in general: a strictly increasing partial measure $f$ on a set $S$ is not always extendible to a strictly positive measure $g$ on a subalgebra $T \supseteq S$. If, however, the set $S$ is closed under addition or under multiplication, and if $T$ is the subalgebra generated by $S$, then the desired extension turns out to be possible (and, as was pointed out in the remarks preceding 1.24 , it can be carried out in exactly one way $\left({ }^{20}\right)$ ).

3 . Countably additive measure.

Definition 3.1. A measure $f$ on a Boolean algebra $A$ is called countably additive if

$$
f\left(\sum_{i<\infty} a_{i}\right)=\sum_{i<\infty} f\left(a_{i}\right)
$$

for every sequence of pairwise disjoint elements $a_{0}, a_{1}, \cdots$ of $A$ provided the sum $\sum_{i<\infty} a_{i}$ of these elements exists.

The Boolean algebra $A$ in 3.1 is not assumed to be countably complete. Usually, however, the notion of a countably additive measure is applied only to countably complete algebras.

As in the case of strictly positive measure, no workable criterion for the existence of a countably additive measure in quite arbitrary Boolean algebras seems to be known. The problem of the existence of such a measure presents sometimes considerable difficulties even when applied to particular, rather simply defined, algebras. Consider, for example, the algebra $B$ of all Borel sets on a bounded interval and an ideal $I$ in $B$ which contains all onepoint sets of $B$ but not all sets of $B$. The answer to the question whether the quotient algebra $B / I$ has a countably additive measure depends on the nature of $I$. If, for instance, $I$ consists of all sets of $B$ of the first category, the algebra $B / I$ has no countably additive measure (see 3.2). If $I$ consists of

(20) The result just mentioned together with some of those stated in $\$ 1$ have certain implications for a class of algebraic systems which is wider than that of Boolean algebras, in fact, for distributive lattices. As is known, every distributive lattice can be imbedded in a Boolean algebra; see MacNeille [1]. Let now $L$ be a distributive lattice with elements 0 and 1 , and let $A$ be an imbedding Boolean algebra. From 1.9 (i) and 1.22 we conclude that (i) every positive modular functional $f$ on $L$ with $f(0)=0$ and $f(1)=1$ can be extended to a measure $g$ on $A$. Assume now, in addition, that $A$ is the smallest Boolean algebra including $L$. We then have, by the remarks made at the end of $\$ 1$, (ii) the extension $g$ is uniquely determined by $f$; and as a particular case of the result just stated in the text, (iii) if $f$ is sharply positive, then $g$ is strictly positive in our sense. This gives another proof of a result in Smiley [1]. For the notions involved in this footnote, see Birkhoff [1]. 
all sets of $B$ of measure $0, B / I$ clearly has a countably additive measure, which in addition is strictly positive. If $I$ is the set of all countable sets of $B, B / I$ has again a countably additive measure but, as is easily seen from 2.4 , no strictly positive measure. In no case, however, does $B / I$ have a twovalued countably additive measure $\left({ }^{21}\right)$.

Consider now the algebra $A$ of all subsets of an uncountable set $U$ and a countably complete ideal $I$ which contains all one-point subsets of $U$ and which is different from $A$. The problem whether there exists a countably additive measure on the quotient algebra $A / I$ depends on the the power of $U$. If the power of $U$ is what is called strongly accessible from $\boldsymbol{\aleph}_{0}$ (if, for instance, its power is $\aleph_{1}$ ), it has been shown that the algebra $A / I$ has no countably additive measure; otherwise the problem remains open. If, however, the power of $U$ is weakly accessible from $\aleph_{0}$ (for example, if its power is c), the algebra $A / I$ is known not to have any two-valued countably additive measure. In the hypothetical case when the power of $U$ is not weakly accessible from $\aleph_{0}$, even the problem of the existence of a two-valued countably additive measure remains open $\left({ }^{22}\right)$.

A rather special class of algebras without a countably additive measure is singled out in the following theorem.

Theorem 3.2. An atomless separable Boolean algebra $A$ has no countably additive measure.

Proof. Suppose $A$ is atomless and separable, and let $f$ be a countably additive measure on $A$. Let $x_{0}, x_{1}, \cdots$ be the elements of a countable set which is dense in $A$, and let $\alpha_{0}, \alpha_{1}, \cdots$ be positive numbers with sum less than 1. For each $n$, choose an element $y_{n} \leqq x_{n}$ with $f\left(y_{n}\right)<\alpha_{n}$ and $y_{n} \neq 0$ (using the atomlessness of $A$ and the countable additivity of $f$ ). Since

it is impossible that

$$
\sum_{n<\infty} f\left(y_{n}\right)<1
$$

$$
\sum_{n<\infty} y_{n}=1
$$

Hence there is an element $x \neq 1$ which includes all the elements $y_{0}, y_{1}, \cdots$. Now $\bar{x} \neq 0$, and for no $n$ is $\bar{x} \geqq x_{n}$, since $x \cdot x_{n} \geqq y_{n} \neq 0$. Thus we have a contradiction to the hypothesis that the elements $x_{n}$ form a set which is dense in $A$.

If $A$ is complete, Theorem 3.2 can easily be derived from results in the

(21) The non-existence of a countably additive measure in $B / I$ in case $I$ consists of all sets of the first category follows from the results in Szpilrajn-Marczewski [1]. The fact that no algebra $B / I$ of the kind considered has a two-valued countably additive measure is stated without proof in Tarski $[4$, p. 165].

(22) For the notions of weak and strong accessibility as well as the result concerning the case of weak accessibility, see Tarski [3, part I, p. 49 and p. 58]. For the case of strong accessibility, see Ulam [1]. 
literature. In fact, as mentioned before (cf. the remarks following 2.5 and those at the beginning of the present section), every complete separable atomless Boolean algebra is isomorphic with the Boolean algebra of Borel sets on a bounded interval divided by the ideal of Borel sets of the first category, and the latter algebra has no countably additive measure.

On the other hand, we can reduce the general case considered in 3.2 to the special case of a complete algebra by using the known fact that every separable atomless Boolean algebra can be imbedded in a complete separable atomless algebra $A^{\prime}$ in such a way that every element of $A^{\prime}$ is a sum of countably many elements of $A\left({ }^{23}\right)$, and by showing that every countably additive measure on $A$ can be extended to a countably additive measure on $A^{\prime}$.

A series of theorems follows which concern two-valued countably additive measures.

TheOREM 3.3. Every Boolean algebra $A$ which is not atomless has a twovalued countably additive measure. Every atomistic Boolean algebra $A$ satisfies the following condition:

(i) For every element $a \neq 0$ in $A$ there is a two-valued countably additive measure $f$ on $A$ with $f(a)=1$.

Proof. If $b$ is an atom of $A$, or an atom of $A$ included in $a$, then, by setting $f(x)=0$ or $f(x)=1$ according as $b \leqq \bar{x}$ or $b \leqq x$, we obtain the desired measure.

Theorems 3.2 and 3.3 together show that, for a separable Boolean algebra to have a countably additive measure, it is necessary and sufficient that $A$ be not atomless. As regards nonseparable Boolean algebras, however, this condition is by no means necessary for the existence of a countably additive measure, and not even for the existence of all two-valued countably additive measures involved in 3.3 (i). In fact, as is easily seen, every countably complete field of sets satisfies 3.3 (i); compare 3.5 below. On the other hand, we can construct countably complete fields of sets which are atomless. For example, let $U$ be the set of all real-valued functions on the set of real numbers; for any given real number $\alpha$, let $F(\alpha)$ be the set of all those functions in $U$ which do not assume $\alpha$ as a value. The smallest countably complete field of sets which contains all the sets $F(\alpha)$ among its elements can be shown to be atomless.

Theorem 3.4. For a Boolean algebra $A$ to satisfy 3.3(i) it is necessary and sufficient that $A$ be isomorphic with a field of sets which is countably complete in the wider sense.

Proof. To show that the condition of our theorem is necessary, we correlate with every element $a \in A$ the set $F(a)$ of all two-valued countably additive measures $f$ with $f(a)=1$. It is easily seen that the family of all sets $F(a)$

${ }^{(23)}$ This can easily be derived from the results quoted in footnote 12 in connection with Statement I. 
thus obtained is a field of sets which is countably complete in the wider sense, and that the function $F$ maps the algebra $A$ on this field isomorphically $\left({ }^{3}\right)$. The proof that the condition in question is sufficient is almost obvious (see the proof of 3.3).

Corollary 3.5. For a Boolean algebra $A$ to be countably complete and to satisfy 3.3 (i) it is necessary and sufficient that $A$ be isomorphic with a countably complete field of sets $\left({ }^{24}\right)$.

Proof. It suffices to remark that every field of sets which is countably complete in the wider sense and which is countably complete as a Boolean algebra must be a countably complete field of sets in the usual sense.

Corollary 3.6. Every Boolean algebra $A$ which satisfies 3.3 (i) is countably distributive.

Proof. Every field of sets which is countably complete in the wider sense is easily seen to be countably distributive. Hence the conclusion follows by 3.4 .

The converse of 3.6 does not hold. In fact, let $U$ be the set of all sets of real numbers, let $A$ be the Boolean algebra of all subsets of $U$, and let $I$ be the ideal of all subsets of $U$ of power c. As was mentioned before, the quotient algebra $A / I$ has no two-valued countably additive measure (the power of $U$ being weakly accessible from $\boldsymbol{\aleph}_{0}$ ); nevertheless, $A / I$ proves to be countably distributive. Thus, $A / I$ provides an example of a countably complete and countably distributive Boolean algebra which is not isomorphic with any countably complete field of sets.

In view of the close connection between two-valued measures and prime ideals (cf. the remarks at the end of $\$ 1$ ), condition 3.3 (i) can be equivalently formulated in terms of prime ideals. With condition 3.3 (i) thus reformulated, 3.4-3.6 can be extended from countably complete and countably distributive Boolean algebras and fields of sets to algebras and fields with higher degrees of completeness and distributivity.

We are now going to discuss measures which are both strictly positive and countably additive. A set of necessary and sufficient conditions for the existence of such a measure-under the assumption that the Boolean algebra concerned is atomless and countably complete-has recently been published; one of these conditions is weak countably distributivity $\left({ }^{25}\right)$. As mentioned

(24) This corollary and the counter-example for Corollary 3.6 given below are to be found in Tarski $[2, \S 16]$.

(25) The set of necessary and sufficient conditions just mentioned is contained in Maharam [1]. The weak countable distributivity of the algebra $L / I$, where $L$ is the field of Lebesgue measurable sets and $I$ the ideal of sets of measure 0 , was previously established in von Neuman [1, part III, pp. $16 \mathrm{ff}$.]; we also find there the proof that the algebra $B / J$, where $B$ is the field of Borel sets and $J$ is the ideal of sets of the first category, is not weakly countably distributive, a result which is a particular case of 3.8 below. 
in that paper, the assumption of atomlessness is not essential. However, for algebras which are not countably complete, not all of these conditions are necessary $\left({ }^{26}\right)$. We wish to show that, at any rate, one of the conditions in question, namely weak countable distributivity, does hold for quite arbitrary algebras.

THEOREM 3.7. Every Boolean algebra $A$ which has a strictly positive countably additive measure is weakly countably distributive.

Proof. Let $a_{i, j}$ be a double sequence of elements of $A$ such that $a_{i, j} \leqq a_{i, j+1}$ for $i, j=0,1, \cdots$. Assume in addition that the product

$$
\prod_{i<\infty} \sum_{j<\infty} a_{i, j}
$$

exists, as well as all the products

$$
\prod_{i<\infty} a_{i, n_{i}}
$$

where $n=\left\langle n_{0}, n_{1}, \cdots\right\rangle$ is any infinite sequence of natural numbers. Let $N$ be the set of all infinite sequences of natural numbers. We put

$$
a_{i}=\sum_{j<\infty} a_{i, j} \text { and } a=\prod_{i<\infty} a_{i}
$$

Consider a strictly positive countably additive measure $f$ on $A$. By the countable additivity of $f$, we have $f\left(a_{i}\right)=\lim _{j \rightarrow \infty} f\left(a_{i}, j\right)$. Hence, given any positive number $\epsilon$, to each $i<\infty$ we can assign an integer $k_{i}$ such that $f\left(a_{i}, k_{i}\right)>f\left(a_{i}\right)$ $-\epsilon / 2^{i+1}$, that is $f\left(a_{i} \cdot \overline{a_{i}, k_{i}}\right)<\epsilon / 2^{i+1}$. Let

$$
d=a \cdot \overline{\prod_{i<\infty} a_{i, k_{i}}}=a \cdot \sum_{i<\infty} \overline{a_{i, k_{i}}} .
$$

It is known that the existence of $\sum_{i<\infty} \overline{a_{i, k_{i}}}$ implies that of $\sum_{i<\infty} a \cdot \overline{a_{i, k_{i}}}$ and the equality

$$
\left.a \cdot \sum_{i<\infty} \overline{a_{i, k_{i}}}=\sum_{i<\infty} a \cdot \overline{a_{i, k_{i}}}{ }^{27}\right)
$$

Hence by (2)

$$
f(d) \leqq \sum_{i<\infty} f\left(a \cdot \overline{a_{i, k_{i}}}\right) \leqq \sum_{i<\infty} f\left(\overline{a_{i} \cdot a_{i, k_{i}}}\right)<\epsilon .
$$

Now suppose $x$ is any element which includes all the products (1) for $n \in N$. Then

${ }^{(26)}$ The following example is due to J. L. Hodges, Jr. Let $A$ be the field of all finite sets of natural numbers and their complements. By $3.8, A$ possesses a strictly positive countably additive measure. However, it is not hard to see that $A$ does not satisfy Maharam's condition II.

(27) See Tarski $[2, \$ 15]$, where further references are given. 


$$
f(x) \geqq f\left(\prod_{i<\infty} a_{i, k_{i}}\right)=f(a)-f(d)>f(a)-\epsilon .
$$

Therefore, by the arbitrary nature of $\epsilon, f(x) \geqq f(a)$. But $a$ and hence $a \cdot x$ includes (1) for all $n \in N$. Therefore $f(a \cdot x) \geqq f(a) \geqq f(a \cdot x)$. By the strictly positive nature of $f, f(a \cdot x)=f(a)$ implies $a \cdot x=a$, or $a \leqq x$. Hence $a$ is the least upper bound of all products (1). A glance at the definition of $a$ completes the proof.

The converse of 3.7 certainly does not hold, for a Boolean algebra which is weakly countably distributive-for example, a countably complete field of sets-may not satisfy the conditions of 2.4. However, by restricting ourselves to separable Boolean algebras, we obtain the following theorem.

THEOREM 3.8. For every separable Boolean algebra $A$ the following conditions are equivalent: (i) $A$ has a strictly positive countably additive measure, (ii) $A$ is atomistic, and (iii) $A$ is weakly countably distributive.

Proof. Condition (i) implies (iii) by 3.7. To derive (i) from (ii), we first assume that the algebra $A$ is infinite. Since it is atomistic and separable, all its atoms can be arranged in an infinite sequence $\left\langle a_{0}, a_{1}, \cdots\right\rangle$ without repetition of terms. We choose any infinite sequence $\left\langle\alpha_{0}, \alpha_{1}, \cdots\right\rangle$ of positive numbers with sum 1 , and we put

$$
f(x)=\sum_{a_{i} \leqq x} \alpha_{i}
$$

for every $x$ in $A$. It is easily seen that the function just defined is a strictly positive countably additive measure on $A$. If $A$ is finite, the same proof, with obvious modifications, applies.

It remains to be shown that (iii) implies (ii). Suppose that $A$ is not atomistic. By definition, there is an element $a \neq 0$ in $A$ which does not include any atom. Let $D$ be a countable set which is dense in $A$, and let $D^{\prime}$ be the set of all elements $d$ of $D$ for which $d \leqq a$. Arrange the elements of $D^{\prime}$ in a sequence $\left\langle d_{0}, d_{1}, \cdots\right\rangle$ without repetitions. It is easily seen that if $a_{i} \neq 0$ is any element $\leqq a$, we can represent $a$ as a sum

$$
a=\sum_{j<\infty} a_{i, j}
$$

with $a_{i, 0}=a_{i}$ and $a_{i, j} \leqq a_{i, j+1}$ for $j=0,1, \ldots$. For each $i<\infty$, we choose such a representation with $a_{i, 0}=a \cdot \overline{d_{i}}$, and note that $d_{i} \cdot \overline{a_{i, j}} \neq 0$ for $j<\infty$. Let $N$ be the set of all infinite sequences $n=\left\langle n_{0}, n_{1}, \cdots\right\rangle$ of natural numbers. We have, for each $n \in N$,

$$
\prod_{i<\infty} a_{i, n_{i}}=0
$$

For otherwise there would be an element $b \neq 0$, and hence a member $d_{k}$ of $D^{\prime}$ 
which is included in all the elements $a_{i, n_{i}}$; but this would imply $d_{k} \leqq a_{k, n_{k}}$, which is a contradiction since $d_{k} \cdot \overline{a_{k, n k}} \neq 0$. Thus the assumption that $A$ is not atomistic implies

$$
a=\prod_{i<\infty} \sum_{j<\infty} a_{i, j} \neq \sum_{n \in N} \prod_{i<\infty} a_{i, n_{i}}=0,
$$

which contradicts (iii). The proof is complete.

Several results stated in this and the preceding sections exhibit close connections between the notions of measurability and that of distributivity of a Boolean algebra. This connection becomes even more striking if we realize that the atomisticity of an algebra can also be treated as a kind of distribtivity; a Boolean algebra is atomistic if and only if it is completely distributive (the definition of the latter notion being obtained by an obvious modification of that of countable distributivity $\left({ }^{28}\right)$ ).

In conclusion we give two theorems on the existence of measures which are not countably additive.

Theorem 3.9. An infinite Boolean algebra has a measure which is not countably additive if and only if 1 can be expressed as the sum of an infinite sequence of pairwise disjoint elements different from $0\left({ }^{29}\right)$.

Proof. Suppose

$$
\sum_{n<\infty} a_{n}=1,
$$

where the elements $a_{n}$ are pairwise disjoint and not equal to 0. Define $f(1)=1$, and $f\left(a_{n}\right)=0$ for $n<\infty$. By $1.10, f$ is a partial measure since no finite sum of elements $a_{n}$ is 1 . By 1.22 there is a measure $g$ on $A$ which agrees with $f$ on the domain of $f$. Obviously, $g$ cannot be countably additive.

Conversely, if 1 is not the sum of an infinite sequence of distinct disjoint elements, then no countable set of disjoint elements not equal to 0 can have a sum, and hence every measure on $A$ is vacuously a countably additive measure.

An example of an algebra which does not satisfy the condition of 3.9 is the algebra of all finite subsets of an uncountable set and their complements.

TheOREM 3.10. Every infinite separable Boolean algebra $A$ has a strictly positive measure which is not countably additive.

Proof. By $2.5, A$ has a strictly positive measure $f$. If $f$ is countably additive, then $A$ must be atomistic by 3.8. In this case, however, the element 1 can clearly be expressed as the sum of the infinite sequence of all the atoms

(28) This is a somewhat stronger form of a joint result of A. Lindenbaum and A. Tarski. For the proof see Birkhoff $[1$, p. 93].

(29) See Tarski $[4$, p. 165]. 
of $A$, and hence, by $3.9, A$ has another measure $g$ which is not countably additive. We put

$$
h(x)=[g(x)+f(x)] / 2
$$

for every $x$ in $A$. The function $h$ just defined is easily seen to be a strictly positive measure on $A$ which is not countably additive.

Regarding the use of transfinite methods in this proof, the remarks following 1.24 apply. We do not know whether Theorem 3.10 could be obtained without the help of such methods even in the simple case when we take for $A$ the algebra of all sets of natural numbers.

\section{BIBLIOGRAPHY}

G. BIRKHOFF

1. Lattice theory, Amer. Math. Soc. Colloquium Publications, vol. 25, 1940.

B. Dushnik ANd E. W. Miller

1. Partially ordered sets, Amer. J. Math. vol. 63 (1941) pp. 600-610.

F. HAUSDORFF

1. Grundzüge der Mengenlehre, Leipzig, 1914.

B. KNASTER

1. Sur une propriêté charactéristique de l'ensemble des nombres réels, Rec. Math. (Mat. Sbornik) N.S. vol. 16 (1945) pp. 281-288.

G. KUREPA

1. Ensemble ordonnés et ramifiés, Publications Mathématiques de l'Université de Belgrade vol. 4 (1935) pp. 1-138.

H. M. MACNEILLE

1. Partially ordered sets, Trans. Amer. Math. Soc. vol. 42 (1937) pp. 416-460.

D. Maharam

1. An algebraic characterization of measure algebras, Ann. of Math. vol. 48 (1947) pp. 154167.

E. W. MILLER

1. A note on Souslin's problem, Amer. J. Math. vol. 65 (1943) pp. 673-678.

A. Mostowski AND A. TARSKI

1. Boolesche Ringe mit geordneter Basis, Fund. Math. vol. 32 (1939) pp. 69-86.

J. voN NEUMANN

1. Lectures on continuous geometry 1936-1937, Princeton, 1937.

W. SIERPIŃSKI

1. Leçons sur les nombres transfinis, Paris, 1928.

2. Sur un problème de la théorie des relations, Annali della $\mathrm{R}$. Scuola normale superiore di Pisa (2) vol. 2 (1933) pp. 285-288.

3. Sur une suite d'ensembles de nombres naturels, Fund. Math. vol. 33 (1945) pp. 9-11.

M. F. SMILEY

1. An extension of metric distributive lattices with an application in general analysis, Trans. Amer. Math. Soc. vol. 56 (1944) pp. 435-447.

M. H. STONE

1. Algebraic characterizations of special Boolean rings, Fund. Math. vol. 29 (1937) pp. 223303.

2. Applications of the theory of Boolean rings to general topology, Trans. Amer. Math. Soc. vol. 41 (1937) pp. 375-481.

3. The theory of representations for Boolean algebras, Trans. Amer. Math. Soc. vol. 39 (1936) pp. 37-111. 
E. SzPILRAJN-MARCZEWSKI

1. Remarques sur les fonctions complètement additives d'ensemble et sur les ensembles jouissant de la propriêté de Baire, Fund. Math. vol. 22 (1934) pp. 303-311.

2. Sur deux propriêtés des classes d'ensembles, Fund. Math. vol. 33 (1945) pp. 303-307.

A. TARSKI

1. Algebraische Fassung des Massproblems, Fund. Math. vol. 31 (1938) pp. 47-66.

2. Cardinal algebras, New York, 1948.

3. Ideale in vollständigen Mengenkörpern, part I, Fund. Math. vol. 32 (1939) pp. 45-63; part II, Fund. Math. vol. 33 (1945) pp. 51-65.

4. Über additive und multiplikative Mengenkörper und Mengenfunktionen, Comptes Rendus des séances de la Société des Sciences et des Lettres de Varsovie, Classe III, vol. 30 (1937) pp. 151-181.

5. Über des absolute Mass linearer Punktmengen, Fund. Math. vol. 30 (1938) pp. 218-234. S. ULAM

1. Zur Masstheorie in der allgemeinen Mengenlehre, Fund. Math. vol. 16 (1930) pp. 140-150.

University of California

Berkeley, Calif. 